\title{
On-sky correction of non-common path aberration with the pyramid wavefront sensor
}

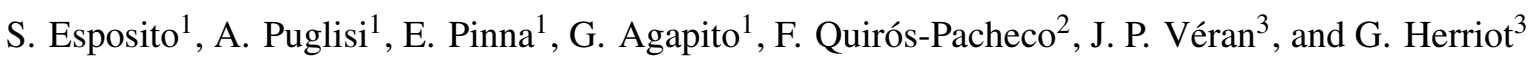 \\ 1 INAF - Osservatorio Astrofisico di Arcetri, Largo E. Fermi 5, 50125 Firenze, Italy \\ e-mail: simone.esposito@inaf.it, guido.agapito@inaf.it \\ 2 GMTO Corporation, 465 N. Halstead St., Suite 250, Pasadena, CA 91107, USA \\ 3 Herzberg Astronomy and Astrophysics, National Research National Research Council of Canada, 5071 West Saanich Road, \\ Victoria, BC V9E 2E7, Canada \\ e-mail: Jean-Pierre.Veran@nrc-cnrc.gc.ca
}

Received 31 October 2019 / Accepted 4 March 2020

\begin{abstract}
The paper deals with with the on-sky performance of the pyramid wavefront sensor-based Adaptive Optics (AO) systems. These wavefront sensors are of great importance, being used in all first light AO systems of the ELTs (E-ELT, GMT, and TMT), currently in design phase. In particular, non-common path aberrations (NCPAs) are a critical issue encountered when using an AO system to produce corrected images in an associated astronomical instrument. The AO wavefront sensor (WFS) and the supported scientific instrument typically use a series of different optical elements, thus experiencing different aberrations. The usual way to correct for such NCPAs is to introduce a static offset in the WFS signals. In this way, when the AO loop is closed the sensor offsets are zeroed and the deformable mirror converges to the shape required to null the NCPA. The method assumes that the WFS operation is linear and completely described by some pre-calibrated interaction matrix. This is not the case for some frequently used wavefront sensors like the Pyramid sensor or a quad-cell Shack-Hartmann sensor. Here we present a method to work in closed-loop with a pyramid wavefront sensor, or more generally a non-linear WFS, introducing a wavefront offset that remains stable when AO correction quality changes due to variations in external conditions like star brightness, seeing, and wind speed. The paper details the methods with analytical and numerical considerations. Then we present results of tests executed at the LBT telescope, in daytime and on sky, using the FLAO system and LUCI2 facility instrument. The on-sky results clearly show the successful operation of the method that completely nulls NCPA, recovering diffraction-limited images with about 70\% Strehl ratio in $\mathrm{H}$ band in variable seeing conditions. The proposed method is suitable for application to the above-mentioned ELT AO systems.
\end{abstract}

Key words. instrumentation: adaptive optics - techniques: high angular resolution

\section{Introduction}

The pyramid wavefront sensor (PWFS) was proposed by R. Ragazzoni in 1996 (Ragazzoni 1996), and today it is successfully used in operations on a few $8 \mathrm{~m}$ class telescopes, such as LBT, Magellan, Keck, and Subaru. The success of the PWFS is closely related to its "non-linear" behavior, exploiting the closed-loop correction of the reference star point spread function (PSF). Qualitatively it is easy to realize that this feature makes the application of sensor signal offsets dependent on closed-loop correction quality. In particular, the correction of non-common path aberration, by using sensor signal offsets, becomes critical and dependent on operating conditions, as shown theoretically in previous works like Korkiakoski et al. (2008a,b), Esposito et al. (2015), and Deo et al. (2018).

The present paper describes a method to apply stable wavefront offsets when working with PWFs. We note here that the method can be applied to other non-linear wavefront sensors like the quad-cell-based Shack-Hartmann (SH) sensor (Hartmann 1900).

The proposed method follows what was initially presented in Esposito et al. (2015) and solves the NCPA application problem by measuring in real time the actual WFS sensitivity, and accordingly updating the reconstructor in use, thus providing stable NCPA correction. The work is organized as follows. In Sect. 2.1 we introduce the system block diagram for a non-linear
WFS using a single scalar optical gain, as originally described in Esposito et al. (2015). The rest of the section generalizes this approach to the case of different sensor modal optical gains and rearranges the block diagram accordingly. In Sect. 2.2 we show how to measure the WFS modal optical gains and provide the mathematical description of a closed-loop control to stabilize their values. Section 3 reports end-to-end numerical simulation results in the case of the Large Binocular Telescope (LBT, Hill 2010) PWFS system (Esposito et al. 2011). In Sect. 4 we report LBT telescope test results using the proposed methods to correct for NCPA between the First Light Adaptive Optics system (FLAO, Esposito et al. 2010a) and LUCI2, the infrared spectroimager of LBT (Seifert et al. 2010, Buschkamp et al. 2012, and Heidt et al. 2018).

\section{Block diagram of a non-linear WFS and its optical gain}

In the following section we introduce a block representation of a non-linear WFS. We then use this representation in a closed-loop diagram to recover the equations governing the $\mathrm{AO}$ system behavior and the corresponding control loop to maintain the NCPA application constant during the course of an observation. 


\subsection{Wavefront sensor optical gains}

The starting point of our work is the consideration that, in general, the WFS response to an incoming aberration is assumed to be linear. This is substantially true for a SH sensor, but it can be quite far off for other WFSs like the PWFS. In this case, the detected signal amplitudes depend on the amplitude of the overall aberration. In other words, the same wavefront aberration will generate different signal amplitudes when it is summed to different wavefront terms. For this reason the PWFS can be defined as a non-linear wavefront sensor. While this is not necessarily a problem in closed-loop operations (provided that the system integrator control gains are optimized in parallel to the loop operations), the non-linear behavior makes the application of pre-calibrated offsets to sensor signals a difficult task. Application of signal offsets is a key aspect of modern AO operations to correct for NCPA between the WFS and the scientific instrument. Without a correct NCPA application the on-sky performance of an AO system can be severely hampered, as shown later in this paper.

In all that follows we assume to be working with an AO loop that uses modal control. We introduce here these main loop elements in general considering upper case letters for matrices and lower case bold letters for vectors:

- the system modal interaction matrix $W_{\mathrm{dl}}$ of dimensions ( $2 N_{\text {sub }}, N_{\text {mod }}$ ), where $N_{\text {sub }}$ and $N_{\text {mod }}$ are the number of subapertures and modes of the considered system. We assume $W_{\mathrm{dl}}$ to be measured in diffraction-limited (DL) conditions or measured by using aberrations of very small amplitude, $\sigma \ll \lambda$, where $\lambda$ denotes the WFS central wavelength;

- a vector $\Delta z$ of differential modal commands of dimension $N_{\text {mod }}$ estimated from the wavefront sensor measurements;

- a vector $z$ of modal correction coefficients so that $\phi_{\text {corr }}=$ $\sum z_{j} B_{j}$, where $B_{j}$ is our orthonormal modal correction basis of modes $B_{j}$;

- a vector of sensor signals $s$ of dimension $2 N_{\text {sub }}$;

- a modal reconstructor $R_{\mathrm{dl}}$ of dimensions $\left(N_{\mathrm{mod}}, 2 N_{\mathrm{sub}}\right)$ obtained as the pseudo-inverse matrix of the matrix $W_{\mathrm{dl}}$ with the property $R_{\mathrm{dl}} \cdot W_{\mathrm{dl}}=I$ with $I$ the identity matrix of dimension $\left(N_{\text {mod }}, N_{\text {mod }}\right)$;

- a diagonal matrix $G$ of integrator transfer functions,

$G(f)=\left(1-e^{-i 2 \pi f T}\right)^{-1} G_{0}$,

where $f$ is the temporal frequency, $T$ is the integration time, and $G_{0}$ is a diagonal matrix of dimensions $\left(N_{\text {mod }}, N_{\text {mod }}\right)$ of integrator modal gains.

In a previous paper (Esposito et al. 2015) we introduced a scalar factor $\gamma_{\text {opt }}$ to deal with the loss of wavefront sensor sensitivity. This factor was multiplied by the diffraction-limited interaction matrix to account for the effect of sensitivity loss in the closed-loop performances of the system and in particular to enable the compensation of the correct amount of noncommon path aberrations. The original block diagram presented in Esposito et al. (2015) is reported in Fig. 1. In this work we generalize the method by replacing the scalar value $\gamma_{\text {opt }}$ with a diagonal matrix $\Gamma_{\text {opt }}$ that takes into account the modal loss of wavefront sensor sensitivity due to a perturbed incoming wavefront. With this assumption we can rewrite the system block diagram of Fig. 1 as shown in Fig. 2.

We discuss now the application of a given NCPA vector $z_{0}$ such that $\phi_{\text {ncpa }}=\sum z_{j} B_{j}$ where the NCPA wavefront $\phi_{\text {ncpa }}$ is decomposed on the AO system modal basis $\left\{B_{j}\right\}$. To simplify the algebra we inject the NCPA by adding an offset to the modal differential commands instead of the common practice of using

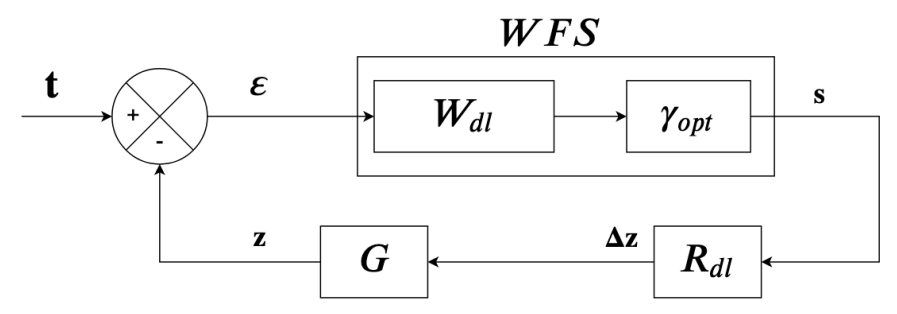

Fig. 1. Original block diagram presented in Esposito et al. (2015). Here $G$ represents the modal integral control defined in Eq. (1) .

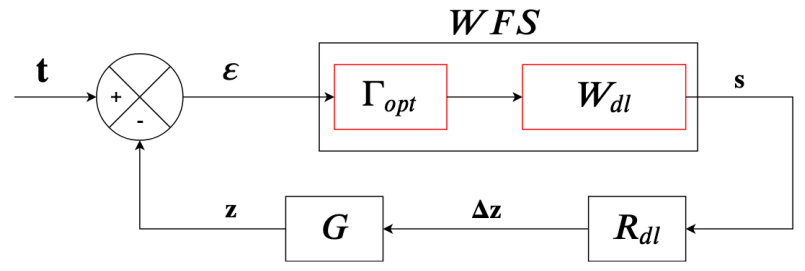

Fig. 2. Block diagram reports the main elements of the considered AO loop. The wavefront sensor block is made up of two parts: $\mathrm{WFS}_{\mathrm{dl}}$ and $\Gamma_{\text {opt }}$. This last time-dependent diagonal matrix takes into account the WFS modal sensitivity variations due to a change in the closed-loop wavefront residuals.

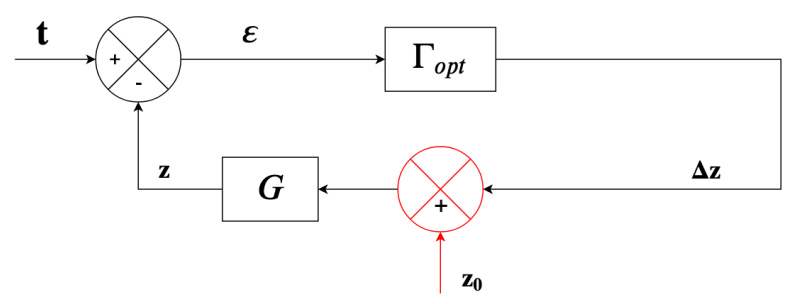

Fig. 3. Block diagram reports the element involved to quantify the resulting modal offset amplitude when a nominal offset amplitude $z_{0}$ is applied to $\Delta \boldsymbol{z}$.

a sensor signal offset vector. This choice leads, in the case of modal correction, to some simplifications. With these assumptions and considering that $R_{\mathrm{dl}} \cdot W_{\mathrm{dl}}=I$ we can rewrite a simplified system block diagram, as reported in Fig. 3.

Using the diagram in Fig. 3, it can be shown (see Appendix A) that the closed-loop modal offset vector achieved, $w_{\text {off }}$, is given by

$w_{\text {off }}=-\Gamma_{\mathrm{opt}}^{-1} z_{0}$,

which is a vector of coefficients that represents the amount of B modes applied on the deformable mirror (DM) in closed-loop. In diffraction-limited conditions $\left(\Gamma_{\mathrm{opt}}=I\right)$ we obtain on the wavefront the required offset $\boldsymbol{\varepsilon}=-\boldsymbol{z}_{0}$. The minus sign in Eq. (2) ensures that $\boldsymbol{w}_{\text {off }}+z_{0}=0$, as required to null the system NCPA $z_{0}$ with the DM offset $\boldsymbol{w}_{0}$. We note that in partial correction regime we get an overcorrection due to the modal optical gain values $\left(\gamma_{\mathrm{opt}}\right)_{j j}<1$. This NCPA correction mismatch happens whenever $\left(\gamma_{\mathrm{opt}}\right)_{j j}$ is different from one and so every time the system is used on sky.

\subsection{Optical gains compensation}

In order to compensate the modal optical gain fluctuations and their effects on NCPA application, we multiply the real-time differential modal correction coefficients vector by a sensitivity 


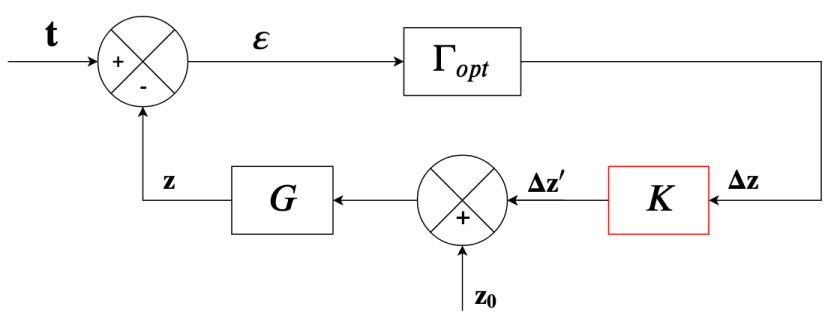

Fig. 4. Diagram of the normalization block introduced to take into account the optical gain changes and correct the amplitudes of the modal coefficients $\Delta \boldsymbol{z}^{\prime}$.

diagonal matrix $K$ in order to bring back the estimated coefficients to diffraction-limited values, obtaining $\Delta z^{\prime}=K \boldsymbol{\Delta} z$.

From the updated block diagram (Fig. 4), we can see that the wavefront offset is now:

$\boldsymbol{w}_{\mathrm{off}}=-K^{-1} \Gamma_{\mathrm{opt}}^{-1} z_{0}$.

Assuming we know the instantaneous value of $\Gamma_{\text {opt }}$, we can simply set $K=\Gamma_{\text {opt }}^{-1}$ at each loop step, restoring the same slope sensitivity used for the system calibration (i.e., diffraction-limited conditions). This allows us to introduce the desired amount of wavefront offset, even during the AO operation when the WFS is operating in partial correction regime. We define the ratio of the offset applied to the desired offset as the NCPA overcompensation factor:

$R_{Z}=\left|w_{\text {off }}\right| /\left|z_{0}\right|$

The condition we are searching for is $R_{Z}=1$. The problem now consists of measuring the diagonal matrix $\Gamma_{\text {opt }}$ or its diagonal elements.

It should be noted here that the considered method has another important effect: it permanently restores the unitary gain of the sensor. Hence, it helps keep the loop optimized and it makes any kind of method that requires a linear WFS or the full knowledge of the sensor sensitivity compatible with the PWFS.

\subsection{Optical gain measurement}

Here we present a method to measure the WFS optical gain $\Gamma_{\text {opt }}$ during an AO observation. This method is an elaboration of previous works using probe signals for the online calibration of $\mathrm{AO}$ system parameters (Véran \& Herriot 2000; Saddlemyer et al. 2004; Esposito et al. 2006; Oberti et al. 2006, and Pinna et al. 2012). We clarify here that the timescale for $\Gamma_{\text {opt }}$ variations are those of the atmospheric conditions variations (e.g., changes in $r_{0}$ or changes in wind speed) so that a suitable sampling rate for this parameter is on the order of a few seconds or more.

In our scheme (see Fig. 5) we add to the integrated modal correction vector $z$ a probe signal, which we choose to be a sinusoidal modulation of each mode $B_{j}$ (of our AO modal basis) at a frequency $\left(f_{\mathrm{p}}\right)_{j}$ and amplitude $\left(z_{\mathrm{p}}\right)_{j}$. In general, the amplitude of this dithering probe can be on the order of $\sim 1-20 \mathrm{~nm}$ RMS, as shown later in the paper, to be negligible for the scientific instrument performance. Moreover, in a real implementation of the techniques, injecting a small number of probe signals allows us to successfully estimate all the optical gains that are a wellbehaved function of the modal radial order (Deo et al. 2018). From the system telemetry we record the temporal history of $z$ (integrated commands plus probe signal) and the differential commands $\boldsymbol{\Delta} \boldsymbol{z}$ obtained from the WFS signals. We introduce

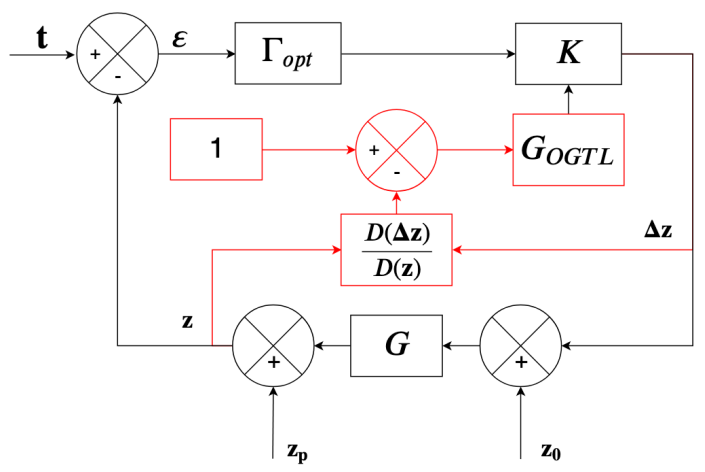

Fig. 5. Complete block diagram describing the proposed method. Highlighted are the two demodulated signals used to derive $\Gamma_{\mathrm{opt}}$ and the modal integral control $G_{\text {OGTL }}$ (defined in Eq. (7)): together they form the optical gain tracking loop (OGTL). Again, the NCPA term is represented as $z_{0}$ (bottom of the figure). The probe signal is injected as $z_{\mathrm{p}}$.

here the operator $D()$ as a demodulation operator of a function $g(t)$ at a frequency $v$ in the time interval $T=1 / v$ defined as

$D(g(t))=\frac{2}{T} \sqrt{\left(\int_{0}^{T} g(t) \cos (2 \pi v t) d t\right)^{2}+\left(\int_{0}^{T} g(t) \sin (2 \pi v t) \mathrm{d} t\right)^{2}}$.

With the considered normalization, the demodulation operator applied to a sinusoidal function $f(t)=A \sin (2 \pi v t)$ gives the result $D(f(t))=A$. We note that in practice to improve the signal-to-noise ratio, more than a single period is used for computation. Demodulating the integral command $z_{j}$ of the mode $j$ at the frequency $v=\left(f_{\mathrm{p}}\right)_{j}$, we can estimate the effective amplitude of the sinusoidal probe signals applied to the AO corrector, filtering out the commands associated with the turbulence correction. In addition, the demodulated signal allows us to take into account the loop dynamic that rejects part of the dithering signal on that particular mode. By demodulating the differential command $\Delta z_{j}$ of the mode $j$, we have an estimate of the probe signal amplitude as measured by the wavefront sensor. The ratio of the two demodulated amplitudes is our estimator for the optical gain correction of the mode $j$. It can be shown (see Appendix A) using the symbols introduced above and block diagram of Fig. 5 that

$\frac{D\left(\Delta z_{j}\right)}{D\left(z_{j}\right)}=k_{j j} \cdot\left(\gamma_{\mathrm{opt}}\right)_{j j}$.

Equations (5) and (3) show that when this ratio is equal to 1 the applied differential offset generates the correct integrated command. To keep this ratio as close as possible to the unitary value, we introduce a closed-loop control named the optical gain tracking loop (OGTL) based on a pure integrator. The inputs of this control loop are the demodulated amplitudes $D\left(z_{j}\right)$ and $D\left(\Delta z_{j}\right)$. The error signal of the OGTL for mode $j$ is given by

$\delta_{j}=1-\frac{D\left(\Delta z_{j}\right)}{D\left(z_{j}\right)}$

Using the pure integrator transfer function written in the Fourier space, the value of $k_{j j}$ matrix element is given by

$k_{j j}=\left(1-e^{-i 2 \pi f T_{\mathrm{OGTL}}}\right)^{-1}\left(\left(G_{0}\right)_{\mathrm{OGTL}}\right)_{j j} \delta_{j}$.

Here $T_{\text {OGTL }}$ and $f$ are the integration time of the OGTL and the temporal frequency, respectively, while $\left(G_{0}\right)_{\text {OGTL }}$ is a diagonal matrix of integrator modal gains $G_{0}$, as defined in Eq. (1). 

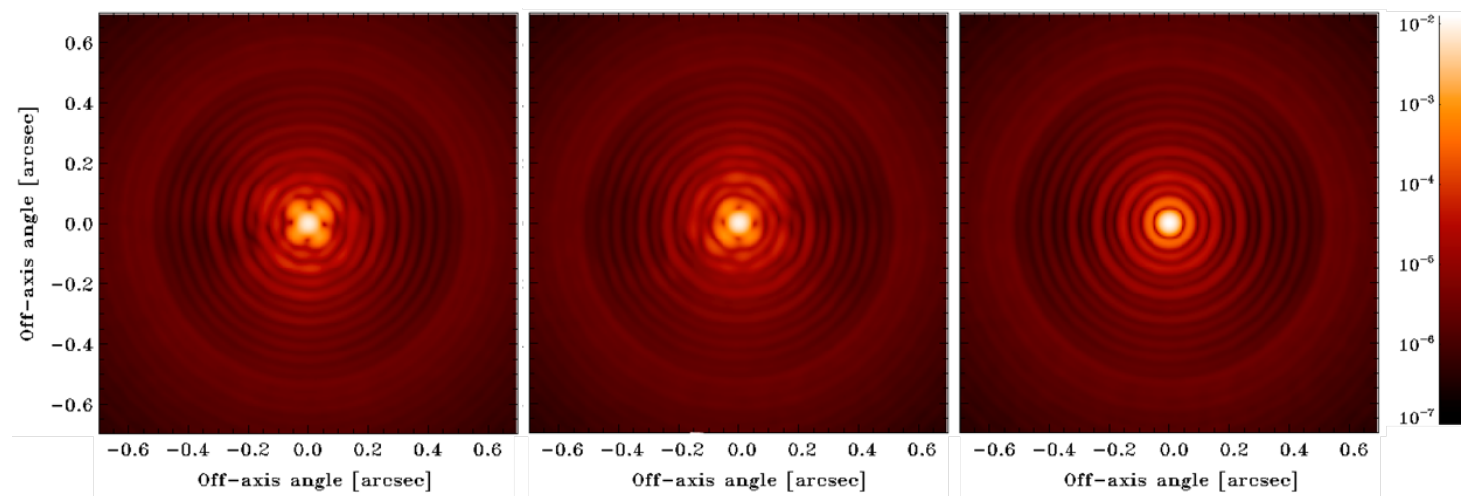

Fig. 6. Simulated long-exposure PSF at $1.6 \mu \mathrm{m}$ on the scientific arm, where NCPA needs to be compensated by the wavefront offset $\boldsymbol{w}_{\text {off }}$. The images are log-view normalized to the peak. Left: no NCPA correction is applied; the PSF shows signs of static aberrations dominated by astigmatism. Center: NCPA correction is applied with OGTL off $\left(k_{\mathrm{opt}}=1\right)$; the NCPA are overcorrected (Fig. 7, top) and static aberration patterns are dimmed but still clear. Right: NCPA correction applied with OGTL active (Fig. 7, bottom); the PSF shows no sign of static aberrations.

The computation of $\delta_{j}$ relies on the demodulation results, so we need to collect a time series of telemetry data from the main AO loop for each step of the OGTL. However, as we mentioned above, the update of the $k_{j j}$ values can be safely done at frequencies below $1 \mathrm{~Hz}\left(T_{\mathrm{OGTL}}>1 s\right)$.

\subsection{Modal space versus signal space slope offset application}

In the described technique, we apply the NCPA correction as offset in wavefront modal space, while, in the past (van Dam et al. 2004; Sauvage et al. 2007; Macintosh et al. 2008, and Vigan et al. 2018), this correction was usually realized applying offsets to the WFS signals. From the point of view of the control loop, the two approaches are equivalent; however, it turns out that the first provides some practical advantages. Given that the modal basis is fixed, the $z_{0}$ remains the same independently of the reconstruction matrix used. As a practical example, offsets in wavefront space do not change with the number of subapertures used. This is a great advantage with PWFS, where the spatial sampling and the tilt modulation are adjusted following the natural guide star (NGS) flux and the seeing conditions. Each combination of spatial sampling and modulation requires a different reconstruction matrix. As a consequence, the NCPA correction in the slope space requires a different set of slope offsets for any different reconstruction matrix, while the modal offset remains the same. The same argument applies to all WFSs where the sensor parameters can be changed, like the SH with weighted center of gravity. Moreover, in the modal command space, the NCPA correction is expressed in wavefront amplitude and can be easily derived from an independent measurement of the system NCPA.

\section{Numerical simulations}

We verified the method described above using end-to-end numerical simulations. We considered the first light AO system (FLAO; Esposito et al. 2010a) of the LBT, featuring a visible light modulated PWFS and an adaptive secondary mirror (ASM; Riccardi et al. 2010) as corrector. We performed the simulations with the PASSATA code (Agapito et al. 2016), updated with the modules needed to introduce the OGTL and NCPA correction, as described in Sect. 2.

In the following we consider that the optical gain matrix $\Gamma_{\mathrm{opt}}$ and the sensitivity matrix $K$ can be written as $\Gamma_{\mathrm{opt}}=I \cdot \gamma_{\mathrm{opt}}$ and $K=I \cdot k_{\mathrm{opt}}$ respectively, with $\gamma_{\mathrm{opt}}$ and $k_{\mathrm{opt}}$ scalar quantities assuming that the sensitivity loss is the same for each wavefront mode. This assumption is supported by the fact that the NCPAs we are dealing with are mostly applied to a few low-order modes having very similar modal optical gains. We consistently use a single probe signal for all modes, and as a consequence the matrix of $\left(G_{0}\right)_{\text {OGTL }}$ gains is reduced to a scalar value $\left(g_{0}\right)_{\text {OGTL }}$. We simulated the presence of NCPAs introducing an aberration pattern on the wavefront after the AO correction and before the PSF computation on the scientific arm. In the reported case, the pattern is composed of $150 \mathrm{~nm}$ RMS of astigmatism and $50 \mathrm{~nm}$ RMS distributed on all modes except tip and tilt with a power law $F^{-2}$, where $F$ represent the spatial frequency. This last component emulates a polishing error on the scientific camera optics. We still represent NCPA with the symbol $z_{0}$. We considered atmospheric turbulence with seeing of $0.8^{\prime \prime}$ and wind speed of $15 \mathrm{~ms}^{-1}$. We set the loop frame rate at $1 \mathrm{kHz}$, correcting 400 modes with a NGS of $m_{R}=8$ (bright regime for the FLAO system; Esposito et al. 2011). We simulated the AO system calibration (interaction matrix) in DL conditions, as performed on the real system at LBT (Esposito et al. 2010b). The probe signal is a $30 \mathrm{~Hz}$ sinusoidal modulation of amplitude $15 \mathrm{~nm}$ (wavefront) applied to mode 5 of the Karhunen-Loève (KL) basis used for the FLAO system control (Quirós-Pacheco et al. 2010). The probe signal is attenuated by a factor $\simeq 0.5$ by the closed-loop running at $1 \mathrm{kHz}$. We note in passing that our KL modes 3, 4, and 5 closely resemble Zernike 5, 4, and 6, respectively.

As a first simulation test, we closed the loop with no NCPA correction to verify the NCPA effect on the PSF at $1.6 \mu \mathrm{m}$ (see Fig. 6, left) and measuring a Strehl Ratio (SR) of 0.56. Then we applied the NCPA without activating the OGTL $\left(k_{\mathrm{opt}}=1\right)$. In Fig. 7, top, we see the temporal evolution of this last case, where the SR curve converges after a few seconds to values around 0.6 to 0.7 (long-exposure average 0.65 ). Due to the partial correction, $\gamma_{\mathrm{opt}}$ is lower than 1 and it is not compensated by $k_{\mathrm{opt}}=1$. We compared $z_{0}$ with the effective wavefront offset applied in the AO loop $\boldsymbol{w}_{\text {off }}$. We verified the collinearity of the two vectors by computing their normalized scalar product, $w_{\text {off }} \cdot z_{0} /\left(\left|w_{\text {off }} \| z_{0}\right|\right)$, which corresponds to 0.998 . This number represents a $z_{0}$ orthogonal component of $\boldsymbol{w}_{\text {off }}$ of $10 \mathrm{~nm}$ amplitude. Then we computed the modulus ratio $R_{Z}=\left|w_{\text {off }}\right| /\left|z_{0}\right|=1.75$. In this case $R_{Z}$ shows the overcompensation of the NCPA by a large factor (1.75). The comparison of $w_{\text {off }}$ with $z_{0}$ is reported in the top plot of Fig. 8. The overcorrection of the NCPA is confirmed by the longexposure PSF in the scientific arm, still showing signs of residual 


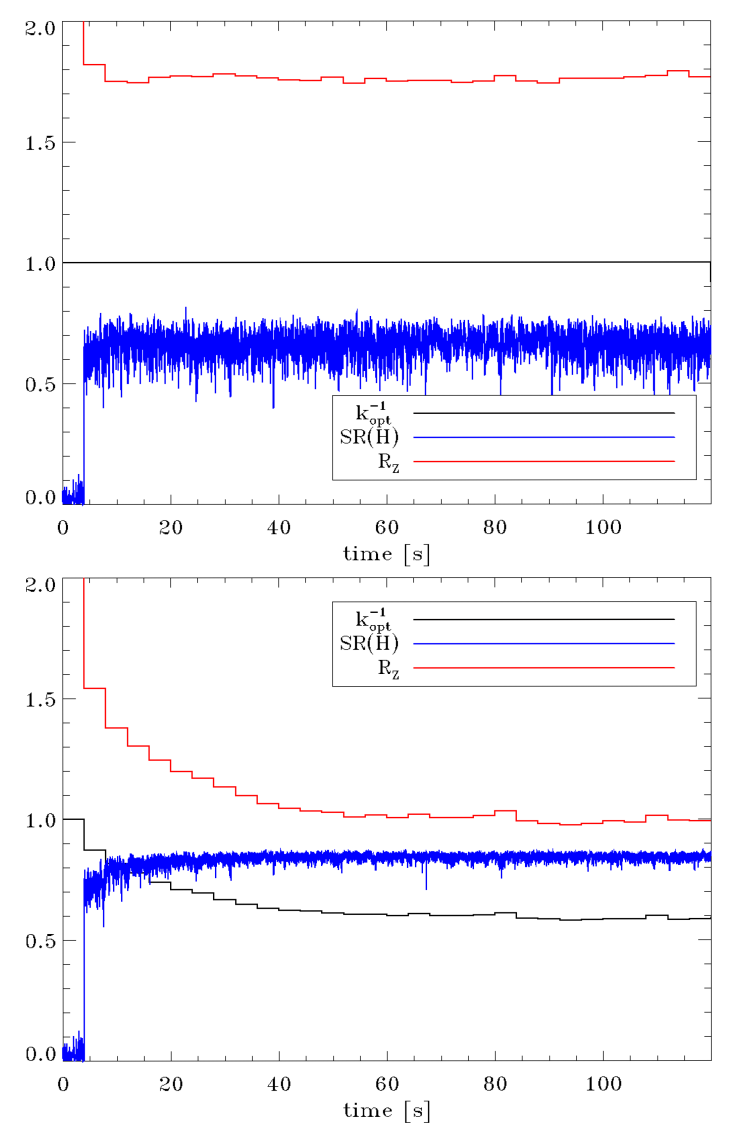

Fig. 7. Results of numerical simulations showing the performance (SR at $1.65 \mu \mathrm{m}$, blue line) when NCPA correction is applied. Top: OGTL is off ( $k_{\text {opt }}$ fixed to 1, black line) and the SR shows values dispersed in the interval $0.5-0.8$, with an average value of $0.67 . R_{z}$ indicates a clear overcorrection of the NCPA. The corresponding long exposure PSF on the scientific arm is shown in the center image of Fig. 6. Bottom: OGTL is active, the SR reaches an average value of 0.87 , and $R_{z}$ converges to 1. The long exposure PSF on the scientific arm is shown in the right panel of Fig. 6.

aberrations (center of Fig. 6). We note here that the 1.75 overcorrection factor is completely consistent with $\gamma_{\mathrm{opt}}=0.65$, where according to Eq. (3) the overcorrection value is the inverse of the optical gain value, namely $1 / 1.75=0.6$, as found from simulations.

After that we again closed the loop, keeping the same parameters and turbulence conditions, while the OGTL was operating at a rate of $0.25 \mathrm{~Hz}$ with $\left(g_{0}\right)_{\mathrm{OGTL}}=0.3$. This combination gives a bandwidth of about $0.02 \mathrm{~Hz}$, suitable for following seeing variation; however, larger bandwidth can be obtained by increasing the OGTL iteration rate, or $\left(g_{0}\right)_{\text {OGTL }}$. The temporal evolution is reported in Fig. 7, bottom. The first measurement is made during the AO loop bootstrap with an initial value of $k_{\text {opt }}=1$. At the second step the OGTL starts compensating with $k_{\text {opt }}>1$. After about ten steps the OGTL converges to $k_{\text {opt }} \sim 1 / 0.6$. The demodulation values are then stable, so our estimation of the optical gain in these conditions is $\gamma_{\mathrm{opt}}=k_{\mathrm{opt}}^{-1}=0.6$. The value of $R_{Z}$ converges to $1 \pm 0.016$, confirming that the AO loop is now introducing the desired amount of wavefront offset, $\boldsymbol{w}_{\text {off }}=z_{0}$, as shown in the bottom plot of Fig. 8. The SR computed at $1.65 \mu \mathrm{m}$ (blue curve) is stable and improved, reaching an average value of 0.843 , almost equal to the case without NCPA, which is 0.848 . The comparison of $\boldsymbol{w}_{\text {off }}$ with $z_{0}$ (bottom of Fig. 8) confirms the
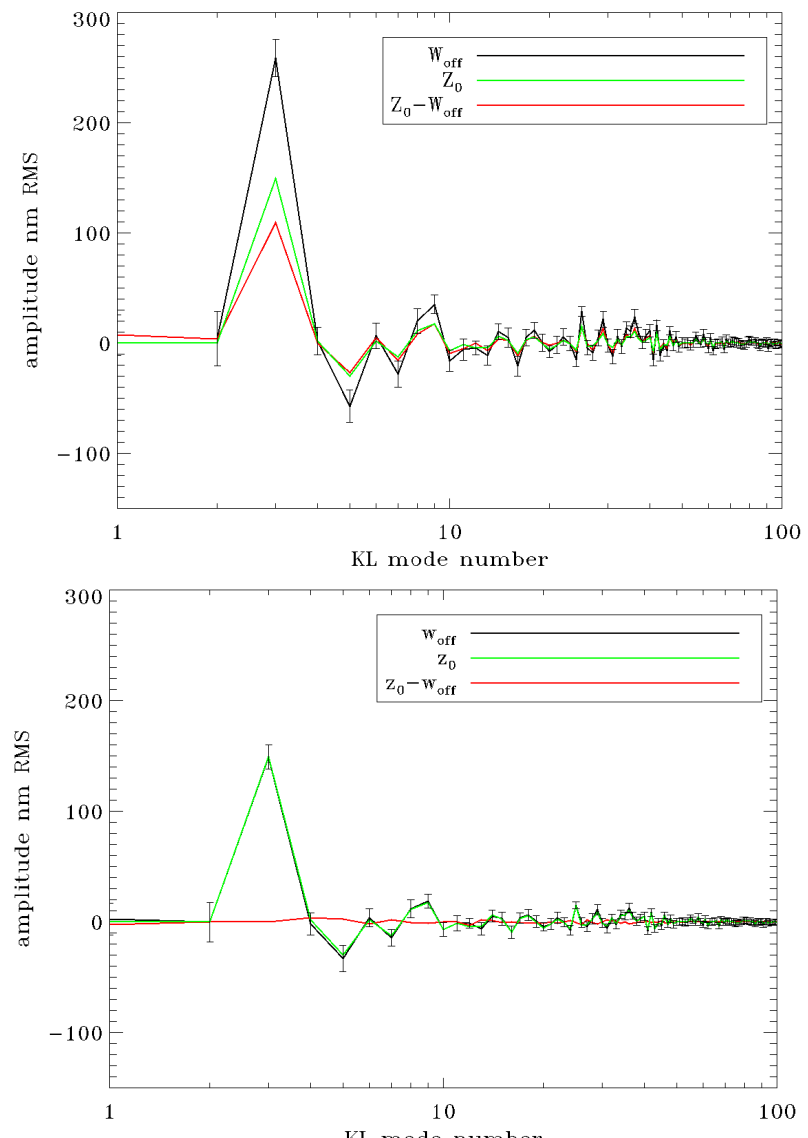

Fig. 8. Comparison of modal amplitudes between the injected offset $z_{0}$ (green line) and that obtained in closed-loop after convergence $\boldsymbol{w}_{\text {off }}$ (black line). When the OGTL is not active (top plot), the injected offset is magnified introducing an overcorrection of NCPA. The OGTL restores the correct offset scaling (bottom plot).

proper scaling of the NCPA correction. Finally, the PSF on the scientific arm (Fig. 6 right) shows no sign of static aberrations.

\section{Experimental results with the FLAO system at the Large Binocular Telescope}

Given the good results obtained with numerical simulations, we moved on to test the technique on the FLAO system at the LBT (Esposito et al. 2010a, 2011), which uses an ASM and a PWFS. The AO system is coupled to the near-IR imager and spectrograph LUCI2, (Seifert et al. 2010, Buschkamp et al. 2012, and Heidt et al. 2018).

Again, as discussed at the beginning of Sect. 3 we consider using a single probe signal and matrices $\Gamma_{\text {opt }}$ and $K$ of the form $\Gamma_{\mathrm{opt}}=I \cdot \gamma_{\mathrm{opt}}$ and $K=I \cdot k_{\mathrm{opt}}$. The above assumption is again based on the fact that measured NCPA for the LUCI2 camera are mainly composed by a few low-order modes, as briefly discussed later in the section. The ASM allows the injection of a time series of actuator positions to be added to the integrator commands during AO loop operations. We used this feature for the creation of the probe signal $z_{\mathrm{p}}$ (see Fig. 5). Moreover, the ASM is provided with an internal metrology system based on calibrated capacitive sensors, one for each actuator; these devices measure the optical surface position with precision of about $1 \mathrm{~nm}$ (Biliotti et al. 1996). We used the time history of the actuator positions for the computation of the $D\left(z_{m}\right)$ term in Eq. (5): we projected the 
actuator positions at each step on the KL modal basis and then we demodulated the amplitude of mode $m$. We note that in the general case of a standard DM with no accessibility to the real mirror positions the demodulation is performed using the closed-loop commands sent to the DM. Demodulating mirror command instead of mirror positions will additionally provide correction for any gain changes between mirror command and mirror position that is not accounted in the system interaction matrix.

In this section we report the results of correction of the NCPA of LUCI2 during the daytime test and on sky during the AO nighttime operation.

\subsection{Telescope daytime test with the calibration source}

The FLAO system can operate in closed-loop in daytime using a calibration source and a retro reflector positioned at the short focus of the ASM. This double-pass optical setup is detailed in Esposito et al. (2010b). As described in that paper, we simulated the atmospheric turbulence injecting commands on the ASM. The test performed in daytime with the calibration source was aimed at verifying in a controlled environment the proper application of the NCPA correction under different seeing conditions, using the internal metrology of the ASM to measure the effective offset applied. In other words, we verified the ability of the method in keeping $\boldsymbol{w}_{\text {off }}=-z_{0}$ under different partial correction conditions.

As an example of NCPA compensation, we considered a coma term (Zernike \#8) with an amplitude of $60 \mathrm{~nm}$. As reported in Table 1 and Fig. 9, we mainly needed three modes (\#9, \#20, and \#37) in our KL basis to reproduce Zernike \#8. During the entire test we ran the AO loop at a frame rate of $1 \mathrm{kHz}$, with a spatial sampling of 30 subapertures across the pupil diameter, and a photon flux provided by the calibration source equivalent to a $m_{R}=8$ reference star. Because the simulated atmospheric disturbance is injected using the DM itself, when the wavefront is corrected by the AO loop, the mirror is kept close to its "zero" position or the applied offset. We recorded the ASM positions during the AO loop operation. For each of the test cases we recorded the telemetry of 24000 loop iterations at a cadence of $500 \mathrm{~Hz}$. The time-averaged position of the mirror provides a direct measurement of the applied wavefront offset.

We injected the atmospheric disturbance closing the loop with and without optical gain tracking loop. We used disturbances equivalent to seeing of $0.8^{\prime \prime}$ and $1.2^{\prime \prime}$, keeping fixed all the AO loop parameters, but optimizing the integrator control gain case by case. We operated the optical gain compensation with a $30 \mathrm{~Hz}$ probe signal of $20 \mathrm{~nm}$ amplitude applied to the $K L$ mode 5 with an OGTL iteration rate of $0.1 \mathrm{~Hz}$ and $\left(g_{0}\right)_{\text {OGTL }}=0.3$. The measured values for $\boldsymbol{w}_{\text {off }}$ are reported in Fig. 9, left, for the case with seeing $0.8^{\prime \prime}$, and Fig. 9, right, for the case at $1.2^{\prime \prime}$. In Table 1 we show the measured modal amplitudes together with the values of $\gamma_{\mathrm{opt}}$. As reported in Sect. 3, the closed-loop attenuates the probe signal by a factor $\simeq 0.5$ when the system runs at the framerate of $1 \mathrm{kHz}$ : the attenuated probe signal is still sufficiently high to work efficiently in the abovedescribed conditions.

When the optical gain tracking loop is off $\left(k_{\mathrm{opt}}=1\right)$, we measured the applied $\boldsymbol{w}_{\text {off }}$ to be 2 to 3 times bigger than $z_{0}$. On the contrary, the optical gain tracking loop was able in both cases to retrieve the proper values of $\boldsymbol{w}_{\text {off }}$ with an accuracy between $3 \%$ and $10 \%$, compatible with the measurement error.
Table 1. Modal amplitudes estimated from time-averaged ASM position telemetry for the different daytime test cases.

\begin{tabular}{ccccc}
\hline \hline \multirow{2}{*}{ Case } & \multirow{2}{*}{$k_{\mathrm{opt}}^{-1}$} & \multicolumn{3}{c}{ Mode amplitude [nm] } \\
\cline { 3 - 5 } & & $\# 9$ & $\# 20$ & $\# 37$ \\
\hline \multirow{2}{*}{$0.8^{\prime \prime}$} & 1 & $-116.3 \pm 12.4$ & $-33.6 \pm 8.0$ & $11.8 \pm 7.6$ \\
& 0.55 & $-63.4 \pm 7.5$ & $-16.4 \pm 5.0$ & $5.9 \pm 3.4$ \\
\hline $1.2^{\prime \prime}$ & 1 & $-150.2 \pm 12.8$ & $-45.3 \pm 8.0$ & $16.6 \pm 6.2$ \\
& 0.40 & $-58.6 \pm 10.7$ & $-17.1 \pm 7.0$ & $6.0 \pm 5.7$ \\
\hline
\end{tabular}

\subsection{On-sky NCPA correction}

Here we present the on-sky results achieved at the LBT telescope using the considered technique to correct for the NCPA between the FLAO system and the LUCI2 N30 diffractionlimited infrared imager of the LBT telescope. We measured the NCPA (limited to up to Zernike \#11) in daytime in DL conditions. In brief, NCPAs are measured in closed-loop, adjusting the modal coefficients offsets to get the best PSF on LUCI2. The measured aberration was dominated by astigmatism $(110 \mathrm{~nm}$ RMS wavefront) with a minor contribution of other low-order modes for a total wavefront RMS of $\sigma_{\mathrm{NCPA}}=140 \mathrm{~nm}$. We projected the measured values on our modal basis obtaining the vector $z_{0}$.

To test the methods on sky we took data during the commissioning of LUCI2 in AO mode, during night of 28-29 January 2015 by closing the loop on a bright star (HD 21038, $\left.m_{R}=6.5\right)$, correcting 400 modes at $1 \mathrm{kHz}$ and under a seeing varying between $0.8^{\prime \prime}$ and $1.3^{\prime \prime}$. We operated the OGTL with a $30 \mathrm{~Hz}$ probe signal of $20 \mathrm{~nm}$ amplitude applied to the $K L$ mode 5 with an iteration rate of $0.1 \mathrm{~Hz}$ and $\left(g_{0}\right)_{\text {OGTL }}=0.3$, as tested in the daytime work. We acquired several images with a narrow filter FeII $(1.65 \mu \mathrm{m})$ with $8 \mathrm{~s}$ integration time, with and without NCPA compensation. We show in the top part of Fig. 10 an example of the images acquired with and without NCPA compensation (left no NCPA compensation, center and right with NCPA compensation), the first two under a seeing of $0.86^{\prime \prime}$ and the third with a seeing of $1.06^{\prime \prime}$. The top left image (image A), top center image (image B), and top right (image C) have a SR of $0.47,0.70$, and 0.58 , respectively. Image A clearly shows the effect of the residual astigmatism of NCPA. On the contrary, images B and C, for which NCPA compensation was applied, have higher SR and round PSF with no astigmatism structures, as shown in detail in the bottom part of Fig. 10. Using the FLAO system telemetry data, we numerically verified the consistency of the achieved results. We use the Maréchal approximation estimating residual wavefront RMS of 226 and $157 \mathrm{~nm}$ RMS for image $\mathrm{A}$ and $\mathrm{B}$ respectively. The SRs of the two images can be compared considering that $\sigma_{\mathrm{A}}^{2}=\sigma_{\mathrm{B}}^{2}+\sigma_{\mathrm{NCPA}}^{2}+\sigma_{\text {res }}^{2}$, where $\sigma_{\mathrm{NCPA}}=140 \mathrm{~nm}$ and $\sigma_{\text {res }}$ is the wavefront RMS difference between the two closed-loop residuals. This last quantity was estimated from the wavefront sensor slopes and has a value of $72 \mathrm{~nm}$. Summing up in quadrature these two terms to the initial $157 \mathrm{~nm}$ rms residual of image B, we obtain for image A a residual estimate of $222 \mathrm{~nm}$, fully compatible with the measured value of $226 \mathrm{~nm}$. Additionally, we can estimate the WF RMS of image $\mathrm{C}$, assuming the same NCPA correction and AO residual scaling with $r_{0}^{-5 / 6}$. Doing this rescaling, we find the image $\mathrm{C}$ residual to be $189 \mathrm{~nm}$ RMS, which compares well with the measured $194 \mathrm{~nm}$ RMS. The above simple error budget computation confirms the correct compensation of NCPA on sky and its stability when seeing changes. We attempted to apply the NCPA 

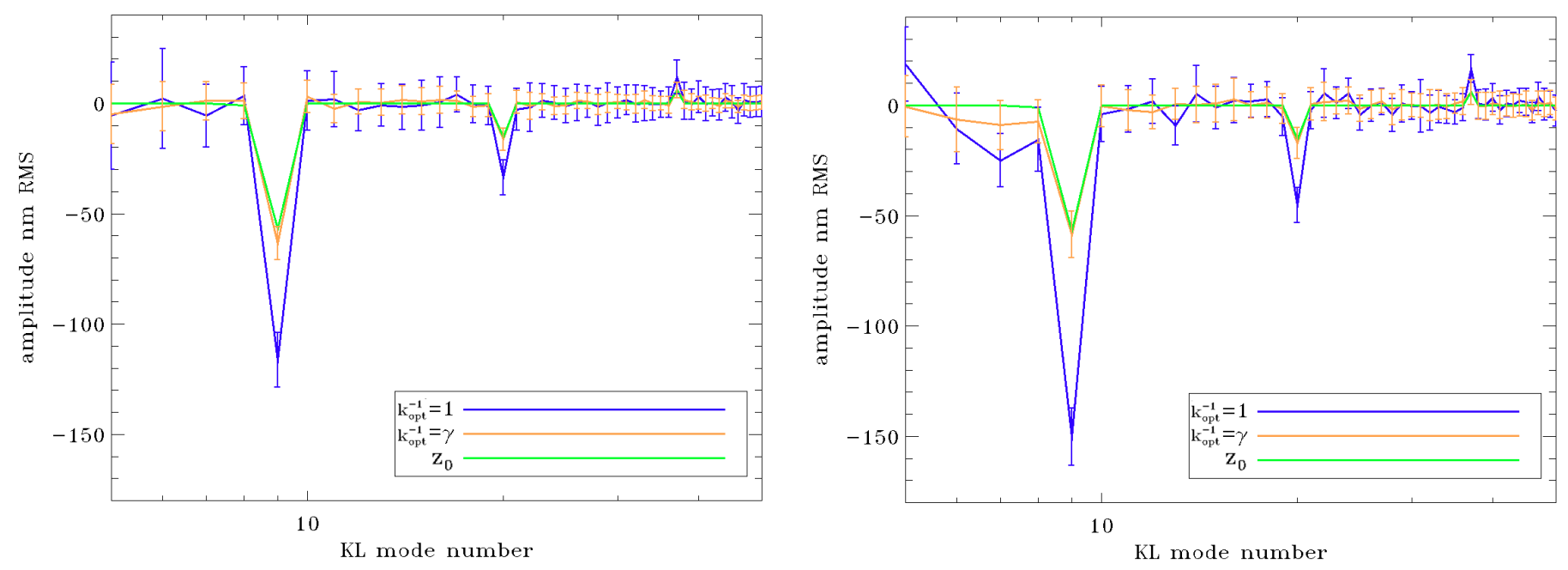

Fig. 9. Injected modal offset $z_{0}$ (green) compared with the average modal amplitude of the DM (wavefront) measured with (orange) and without (blue) optical gain correction. Left: case with injected disturbance equivalent to $0.8^{\prime \prime}$ of seeing. The lower modes (1-4) are not reported, being affected by the correction of the optical system alignment drifts. Right: case with injected disturbance equivalent to $1.2^{\prime \prime}$ of seeing.
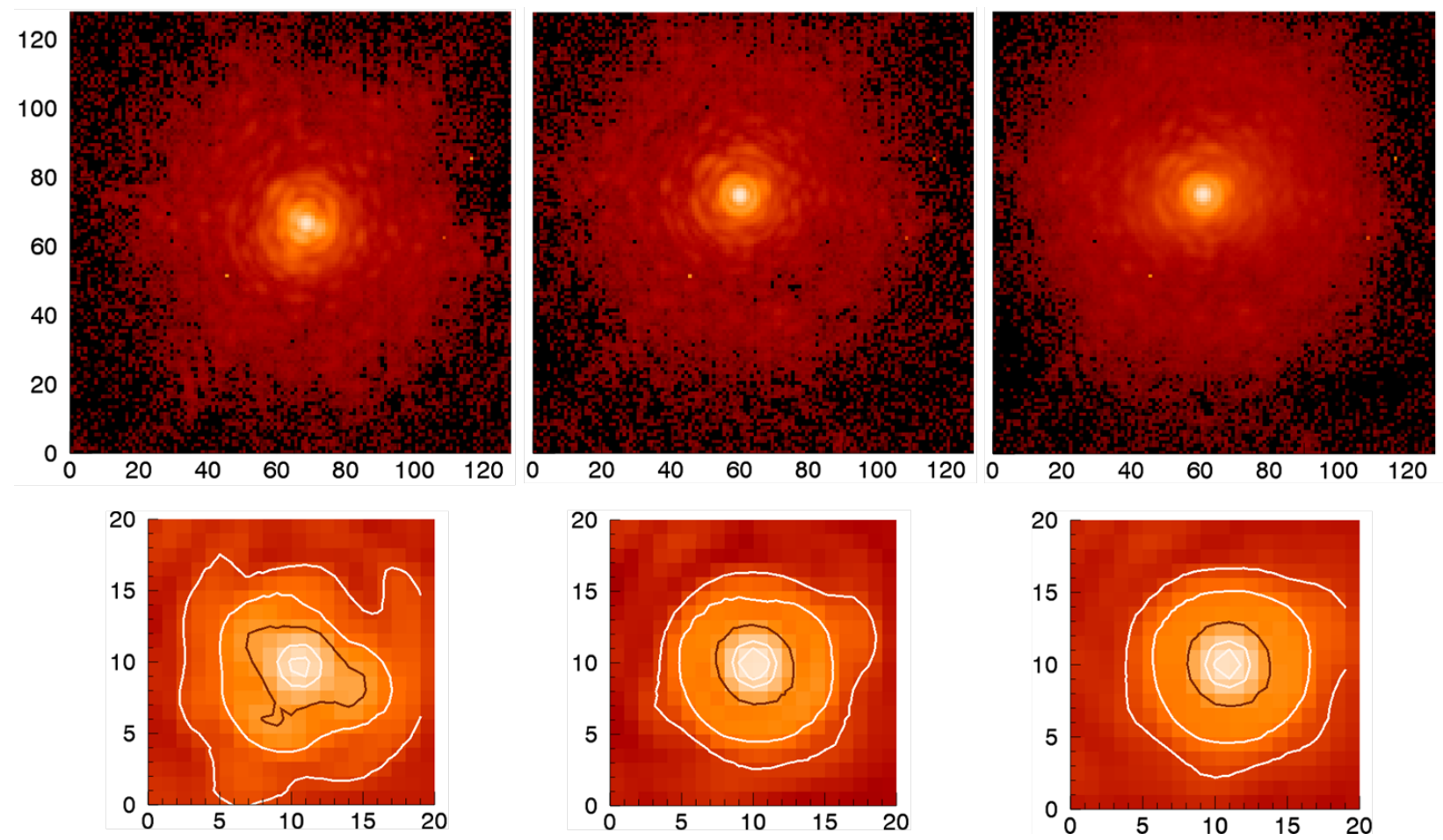

Fig. 10. Image in logarithmic scale of a bright star used as AO reference and imaged on the LUCI N30 camera $\left(\right.$ plate scale $=15 \mathrm{mas}^{-1}$ ) at $1.65 \mu \mathrm{m}$. The three images (A), (B), and (C), from left to right, show the full PSF in a field of 1.9 arcsec; the three bottom images show the detail on the central peak with contour lines underlining low-order aberrations. Image $(A)$ : the NCPA correction is not applied and the seeing is 0.86 arcsec; the first diffraction ring shows lobes and the SR is 0.47. Image $(B)$ : the NCPA correction is applied with optical gain loop control and the seeing is still 0.86 arcsec, the first ring is round, and SR increased to 0.70. Image $(C)$ : the NCPA correction is applied with optical gain loop control and the seeing is 1.06 arcsec; the first ring is still round and the SR is 0.58 .

correction without optical gain compensation, but the magnified NCPA correction made the AO loop unstable and the closed-loop operation impossible.

The described technique is currently implemented in the FLAO system to work with LUCI1 and LUCI2 imagers and it is routinely used in diffraction-limited observations at LBT. As an example of successful performance during science operations, we report in Fig. 11 a subfield of the Palomar10 globular cluster comparing the PSF quality, with and without NCPA correction.

\section{Conclusions}

We presented the theory and application of a method to correct NCPA in AO systems using non-linear wavefront sensors like the PWFS. The considered theory shows how NCPA application is impacted by the changes in the PWFS sensitivity that is accounted for using a modal optical gain diagonal matrix $\Gamma_{\text {opt }}$. The use of time-varying sinusoidal probe signals ( $\sim 10 \mathrm{~nm}$ RMS) injected in the system allows us to measure the elements of 

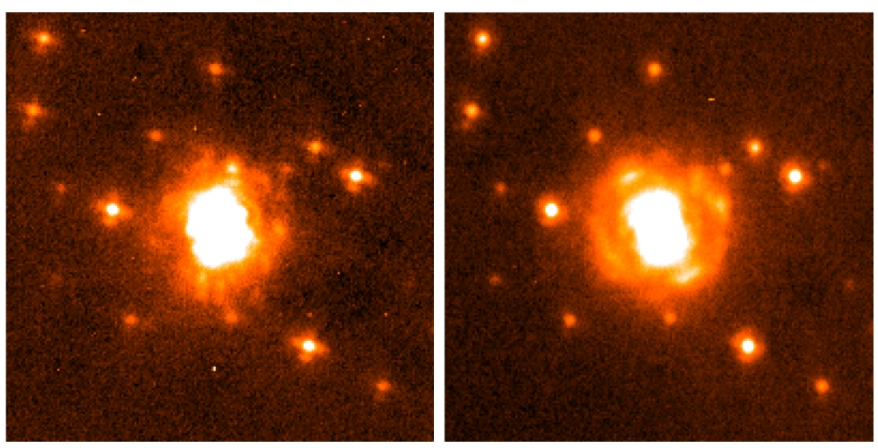

Fig. 11. Example of NCPA correction for an AO observation of Palomar10 globular cluster. We compare a subfield of $3^{\prime \prime} \times 3^{\prime \prime}$ on the LUCI2 N30 frames with (right) and without (left) NCPA correction. The images clearly show the improvement in PSF quality. The images are $K_{s}$ band with total exposure time of $105 \mathrm{~s}$ and $960 \mathrm{~s}$ for left and right, respectively.

the matrix $\Gamma_{\text {opt }}$, the modal optical gains. The knowledge of this matrix enables the application of the proper NCPA offset values. Numerical simulation of the FLAO system at the LBT, assuming the same value $\gamma_{\mathrm{opt}}$ is used for all modal optical gain (i.e., $\left.\Gamma_{\mathrm{opt}}=I \cdot \gamma_{\mathrm{opt}}\right)$ showed that the method provides efficient NCPA compensation of more than 150nm RMS. Experimental results at the LBT telescope, both in daytime and on-sky operations, again in the simplified assumption stated above, clearly show that the proposed technique provides accurate and stable application of wavefront offsets. The technique significantly improves the SR of AO-corrected on-sky images, in the reported case from 0.35 to 0.7 in $H$ band, removing all signatures of astigmatism in the acquired PSFs irrespective of the seeing variation. The method is routinely used at LBT providing diffraction-limited observation with LUCI.

This method is directly applicable to all PWFS based AO systems, including those in the design phase for the next generation of giant ground-based telescope E-ELT, GMT, and TMT.

Acknowledgements. The authors would like to acknowledge the support given to development of this technique provided by the LBT AO group and LBT astronomers. Moreover, the authors acknowledge the support from the LUCI instrument team lead by J. Heidt and W. Seifert in developing LUCI science observation with $\mathrm{AO}$ and $\mathrm{G}$. Fiorentino for providing the Palomor10 images.
Finally the authors want to thank an unknown referee for the insightful and constructive comments that contributed to a better structured paper.

\section{References}

Agapito, G., Puglisi, A., \& Esposito, S. 2016, in Adaptive Optics Systems V, Proc. SPIE, 9909, 99097E

Biliotti, V., Biasi, R., Brusa, G., et al. 1996, European Southern Observatory Conference and Workshop Proceedings , 54, 213

Buschkamp, P., Seifert, W., Polsterer, K., et al. 2012, in Ground-based and Airborne Instrumentation for Astronomy IV, Proc. SPIE, 8446, 84465L

Deo, V., Gendron, É., Rousset, G., Vidal, F., \& Buey, T. 2018, in Adaptive Optics Systems VI, Proc. SPIE, 10703, 1070320

Esposito, S., Tubbs, R., Puglisi, A., et al. 2006, in Advances in Adaptive Optics II, Proc. SPIE, 6272, 62721C

Esposito, S., Riccardi, A., Fini, L., et al. 2010a, in Adaptive Optics Systems II, Proc. SPIE, 7736, 773609

Esposito, S., Riccardi, A., Quirós-Pacheco, F., et al. 2010b, Appl. Opt., 49, G174 Esposito, S., Riccardi, A., Pinna, E., et al. 2011, in Astronomical Adaptive Optics Systems and Applications IV, Proc. SPIE, 8149, 814902

Esposito, S., Puglisi, A., Pinna, A., et al. 2015, Adaptive Optics for Extremely Large Telescopes 4 - Conference Proceedings

Hartmann, J. 1900, Naturwissenschaften, 20, 17

Heidt, J., Pramskiy, A., Thompson, D., et al. 2018, in Ground-based and Airborne Instrumentation for Astronomy VII, Proc. SPIE, 10702, 107020B

Hill, J. M. 2010, Appl. Opt., 49, 115

Korkiakoski, V., Vérinaud, C., \& Le Louarn, M. 2008a, in Adaptive Optics Systems, Proc. SPIE, 7015, 701554

Korkiakoski, V., Vérinaud, C., \& Louarn, M. L. 2008b, Appl. Opt., 47, 79

Macintosh, B. A., Graham, J. R., Palmer, D. W., et al. 2008, in Adaptive Optics Systems, Proc. SPIE, 7015, 701518

Oberti, S., Quirós-Pacheco, F., Esposito, S., et al. 2006, in Advances in Adaptive Optics II, Proc. SPIE, 6272, 627220

Pinna, E., Quirós-Pacheco, F., Riccardi, A., et al. 2012, in Adaptive Optics Systems III, Proc. SPIE, 8447, 84472B

Quirós-Pacheco, F., Busoni, L., Agapito, G., et al. 2010, in Adaptive Optics Systems II, Proc. SPIE, 7736, 77363H

Ragazzoni, R. 1996, J. Mod. Opt., 43, 289

Riccardi, A., Xompero, M., Briguglio, R., et al. 2010, in Adaptive Optics Systems II, Proc. SPIE, 7736, 77362C

Saddlemyer, L. K., Herriot, G., Véran, J. P., Smith, M., \& Dunn, J. 2004, in Advancements in Adaptive Optics, Proc. SPIE, 5490, 1384

Sauvage, J.-F., Fusco, T., Rousset, G., \& Petit, C. 2007, J. Opt. Soc. Am. A, 24, 2334

Seifert, W., Ageorges, N., Lehmitz, M., et al. 2010, in Ground-based and Airborne Instrumentation for Astronomy III, Proc. SPIE, 7735, 77357W

van Dam, M. A., Mignant, D. L., \& Macintosh, B. A. 2004, Appl. Opt., 43, 5458

Véran, J. P., \& Herriot, G. 2000, in Adaptive Optical Systems Technology, Proc. SPIE, 4007, 642

Vigan, A., N'Diaye, M., Dohlen, K., et al. 2018, in Adaptive Optics Systems VI, Proc. SPIE, 10703, 1070350 


\section{Appendix A: Closed loop mesh equations}

We report here the derivation of Eqs. (2)-(5) that are fundamental to the method. We recall here that a method for monitoring the optical gain value is essential for efficient NCPA compensation with the PWFS or a non-linear WFS.

To derive Eq. (3) we refer to the block diagram of Fig. 4 and write the following mesh equations valid for any given temporal frequency:

$z=G \cdot \Delta z$

$\Delta z=K \cdot \Gamma_{\mathrm{opt}} \cdot \varepsilon+z_{0}$

$\varepsilon=t-z$

where $\varepsilon$ is the closed-loop residual in the modal space considered. Replacing the first two equations above and replacing into Eq. (A.3) we obtain

$\boldsymbol{\varepsilon}=\boldsymbol{t}-G \cdot\left(K \cdot \Gamma_{\mathrm{opt}} \cdot \boldsymbol{\varepsilon}+z_{0}\right)$.

We recall that in the above equation the $\mathrm{G}$ matrix coefficients are a function of the frequency and accounts for the integral control as shown in Eq. (1), and so $G(f=0)=\infty$. Hence, we find

$K \cdot \Gamma_{\text {opt }} \cdot \boldsymbol{\varepsilon}+z_{0}=0$.

So we find for a static closed-loop residual:

$\boldsymbol{\varepsilon}=-\Gamma_{\mathrm{opt}}^{-1} \cdot K^{-1} \cdot z_{0}$.

Equation (2) can be easily derived by replacing $K$ with the identity matrix.

To derive Eq. (5) we refer to the block diagram of Fig. 5 and write the following mesh equations:

$z=G \cdot \Delta z+z_{\mathrm{p}}$.

Combining Eqs. (A.7) and (A.2), to single out $z$ we get

$z+G \cdot K \cdot \Gamma_{\mathrm{opt}} \cdot z=G \cdot K \cdot \Gamma_{\mathrm{opt}} \cdot t+G \cdot z_{0}+z_{\mathrm{p}}$.
Applying the demodulation operator for $f=\left(f_{\mathrm{p}}\right)_{j}$ to both terms of the above equation and considering $D(v)=\left\{v\left(f_{\mathrm{p}}\right)_{j}\right\}, j=$ $\left[1, n_{\text {mod }}\right]$ we get

$D(z)+G\left(f_{\mathrm{p}}\right) \cdot K \cdot \Gamma_{\mathrm{opt}} \cdot D(z)=\left|z_{\mathrm{p}}\right|$

and

$D(z)=\left(I+G\left(f_{\mathrm{p}}\right) \cdot K \cdot \Gamma_{\mathrm{opt}}\right)^{-1} \cdot\left|z_{\mathrm{p}}\right|$.

Combining Eqs. (A.2) and (A.7), to single out $\Delta z$ we get

$\Delta z=K \cdot \Gamma_{\mathrm{opt}} \cdot \boldsymbol{t}-G \cdot K \cdot \Gamma_{\mathrm{opt}} \cdot \Delta z-K \cdot \Gamma_{\mathrm{opt}} \cdot z_{\mathrm{p}}+z_{0}$.

Applying the demodulation operator to both terms of the above equation we get

$D(\boldsymbol{\Delta} \boldsymbol{z})=G\left(f_{\mathrm{p}}\right) \cdot K \cdot \Gamma_{\mathrm{opt}} \cdot D(\boldsymbol{\Delta} \boldsymbol{z})+K \cdot \Gamma_{\mathrm{opt}} \cdot\left|z_{\mathrm{p}}\right|$,

and so

$D(\boldsymbol{\Delta z})=\left(I+G\left(f_{\mathrm{p}}\right) \cdot K \cdot \Gamma_{\mathrm{opt}}\right)^{-1} \cdot K \cdot \Gamma_{\mathrm{opt}} \cdot\left|z_{\mathrm{p}}\right|$.

Then, noticing that all matrices in Eq. (A.13) and (A.12) are diagonal, we can derive the $j$ th elements of the considered vectors $D(\Delta z)$ and $D(z)$ as

$D\left(z_{j}\right)=\frac{\left|\left(z_{\mathrm{p}}\right)_{j}\right|}{1+g_{j j}\left(f_{\mathrm{p}}\right) \cdot k_{j j} \cdot\left(\gamma_{\mathrm{opt}}\right)_{j j}}$

$D\left(\Delta z_{j}\right)=\frac{k_{j j} \cdot\left(\gamma_{\mathrm{opt}}\right)_{j j} \cdot\left|\left(z_{\mathrm{p}}\right)_{j}\right|}{1+g_{j j}\left(f_{\mathrm{p}}\right) \cdot k_{j j} \cdot\left(\gamma_{\mathrm{opt}}\right)_{j j}}$.

Hence, the ratio between $D\left(\Delta z_{j}\right)$ and $D\left(z_{j}\right)$ is given by

$\frac{D\left(\Delta z_{j}\right)}{D\left(z_{j}\right)}=\frac{k_{j j} \cdot\left(\gamma_{\mathrm{opt}}\right)_{j j} \cdot\left|\left(z_{\mathrm{p}}\right)_{j}\right|}{\left|\left(z_{\mathrm{p}}\right)_{j}\right|}=k_{j j} \cdot\left(\gamma_{\mathrm{opt}}\right)_{j j}$,

providing the derivation of Eq. (5). 